\title{
The Simulation and Analysis of Modified LEACH Protocol using TOSSIM in Wireless Sensor Networks
}

\author{
Raju Dutta \\ Narula Institute of Technology \\ Dept. of Mathematics \\ Kolkata, West Bengal
}

\author{
Shishir Gupta \\ Indian School of Mines \\ Dept. of Applied Mathematics \\ Dhanbad, Jharkhand
}

\author{
Debraj Paul \\ Narula Institute of Technology \\ Computer Science and \\ Engineering \\ Kolkata, West Bengal
}

\begin{abstract}
The most important routing protocol LEACH of wireless sensor networks (WSNs) has been incorporated by W. Heinzelman. LEACH uses the concept of dynamic clustering when sensor nodes are deploying randomly where number of cluster impact on the network. This paper describes the network quality that depends on the optimum selection of number of clusterhead as a Modification on LEACH protocol using new operating system TOSSIM. In this paper we discussed and explain the comparison of data packet broadcasting, energy consumption and dead node of LEACH and Modified LEACH respectively. Simulation result shows Modified LEACH performs better than LEACH for extending overall network performances.
\end{abstract}

\section{Keywords}

Modified LEACH protocol; TinyOS; nesC; TOSSIM; Wireless Sensor Network.

\section{INTRODUCTION}

WSN is very much popular in the military environment, mines and other hazards in the environment to detect the stability. Therefore WSNs has huge use in industry, military, corps, human care and so on. The routing protocols are very much important topics in network. Tiny sensors have been developed with new information technology for easy data processing, data aggregation and effective communication in the wireless network. Because WSNs can give more better result of the environment efficiently at any moment. Wireless sensor network gather different types of data information from the environment and transmit data directly to the user from the sensors to embedded computing, processing and communication technology [1].

Wireless sensor networks (WSNs) consists sensors which communicate to sensors by multihop. Sensor, sensing object and observer form the three factors in WSNs [1][2][3]. WSNs protocol stack contains physical layer, data link layer, network layer, transport layer and application layer [4]. According to network architecture, routing protocols are generally classified as plane routing, grade routing, and position routing. LEACH (Lower Energy Adaptive Clustering Hierarchy) protocol is a grade routing protocol. Paper [1] and [5] introduce LEACH protocols in detail. Paper [6] presents a modified protocol, but it is still faulty and the performance matrixes are complex and Multi-hop protocol is studied in paper [7], but it is quite complex.

As the power of the sensor node can not be increased then how the nodes can be efficiently use in the network so that system energy becomes the prime factor for designing routing protocol. LEACH protocol has been considered as layered protocol for WSNs, which uses random clustering formation [1]. This routing protocol is different than other protocol for low energy consumption and prolonging system lifespan. But the number of cluster is grate problem in the network which depends on number of clusterhead, so it is important to know optimum clusterhead to perform the network better. In this paper, new protocol for energy consumption is presented using TOSSIM and several comparisons have been presented.

\section{LEACH PROTOCOL}

\subsection{Introduction of WSNs}

Wireless sensor networks is a subset of ad-hoc network consists static or dynamic nodes for data transmission in the network by self organized way and multi hop way. The main activity of node is datacentric and collecting information for the user [8].. It is seen that sensor node performing mainly three functions data acquisition, processing and transmission, which are related to wireless technology, modern technology, computer and communication technology. Here the sensors are used as a basic elements of WSNs where sensors are collecting, processing and transmitting information.

\subsection{Cluster Head selection algorithm}

Two phases are in LEACH protocol which are (i) the cluster formation and (ii) data receiving and transmission phase and round as defined the time slot gap between two phases. In the cluster head selection phase sensor node generates a random number which lies within 0 and 1 , if that number is less than threshold value $\mathrm{T}(\mathrm{n})$ then in that round it selects that node to act as clusterhead, and acknowledge that node to the other neighbor [9]. The formula for $\mathrm{T}(\mathrm{n})$ is given below:

$$
T(n)= \begin{cases}\frac{p}{1-p^{*}\left(r \bmod \frac{1}{p}\right),} & \text { if } n \in G \\ 0 & \text { otherwise }\end{cases}
$$

$r$ indicates the present round, selection of a node to be a clusterhaed with probability, $p$ and those nodes are still not become clusterhead in previous ( $\mathrm{r}-1)$ round, will form another set $G$. We consider here $N$ as the total number of nodes in the network, the approximate number of clusterhead to be considered as $k$, where $p=k / N$. Each node has opportunity to become a clusterhead once by this algorithm, if one node become clusterhead in a particular round, it will lose to become another time clusterhead in other round.

\section{LEACH PROTOCOL ANALYSED USING TOSSIM \\ 3.1 TinyOS and nesC}

TinyOS [10] is an open source component based operating system designed for wireless sensor networks. It features a 
component-based architecture which enables rapid innovation and implementation while minimizing code size as required by the severe memory constraints inherent in sensor networks. The TinyOS system, libraries, and applications are written in nesC, a dialect of $\mathrm{C}$ programming language. The nesC supports the TinyOS concurrency model and its programs are a set of software components which are connected to each other using interfaces. A nesC [11] application consists of components which can use or provide interfaces and different components are connected using these interfaces. An interface in a nesC application consists of commands and events. A component which provides the interface has to provide the implementation for the commands in that interface and can signal the events to the components using that interface. Otherwise, a component using the interface which has to provide the implementation for the event handlers in the interface. Modules in TinyOS provide the implementation of the components and the configuration. A scenario is a collection of components and the wiring between the interfaces of these components which describes the complete application. NesC programs are built out of software components some of which are hardware abstractions. TinyOS provides interfaces and components for common abstractions such as packet communication, routing and sensing. The framework developed in this paper, provides the user with higher level abstractions with some generic services so that they can be directly used. It also aims at providing the user a global view of application while abstracting the underlying communication details. TOSSIM is nesC based language. In nesC application a component using provides and interfaces. An interface generally models some services in nesC. They contain commands and events. To execute the operation commands request i.e sending some request for job such as message.send() this command function is sending temp component to Main Component through StdControl interface. In advertisement phase in Fig. 1it request neighbor node to act as a clusterhead in LEACH, send data by command sendMsg.dend() and toggle red LED to indicate transmit data by command led.redToggle(). In this phase one node invites other nodes in the neighbor table to accept the broadcast massage and act as a clusterhead. In Fig. 2 Modified LEACH select clusterhead and increasing hopcount to send data at distination.

\subsection{Simulation Parameter}

Set monitoring area according to the Motes or default system generated area for simulation, the number of nodes can varies according to the need, hopcount varies with nodes, base station can be considered according to the user. TinyViZ shows the movement of communication from node to node.

\subsection{Modified LEACH Analysis Method}

Hare we considered a random system generated topology in TOSSIM. This paper uses some interface command for simulation. Commands are typically request to execute an operation i.e. sending some request for job such as message.send () this command function is sending temp component to Main Component through StdControl Interface. But event is the reply for this job which is sending through this same StdControl interface from Main to temp.like send.sendDone() event function. RouteFlood component is connect to Main component through StdControl Interface (Provides Interface). RouteFlood sending the some commend message to Main and the Main also sending an reply message for this request. Actually this diagram show the visualization part of the wiring technology, which is in the configuration part.

\subsection{Modified LEACH Simulation and Analysis}

Energy consumption of the network under various number of clusterhead for LEACH and Modified LEACH protocol has been observed, from simulated result it is observed that optimum number of clusterhead and the number of clusters in the WSNs to obtained energy consumption minimum. Then Modified LEACH protocol is proposed and analyzed from different aspect of network through energy consumption with different nodes and the statistics of receiving of data by the base station computed. Thus by simulation result it shows Modified LEACH protocol performs better than LEACH under optimum clusterhead.

\subsubsection{Simulation of Clusterhead}

In Fig. 3 it is seen in that LEACH forms only two cluster head, which ensures energy consumption will increase when node transmitting data to clusterhead, so the cumulative power lose per round will be more. Forming very less number of clusterhead consumes more power in the network because more nodes will be involved to send data to the base station. Energy consumption will increase proportionally with clusterhead upto certain level, and the network efficiency reduces. Considering 100 nodes we simulated LEACH and Modified LEACH both and received 14 nodes inside one cluster for LEACH and 8 nodes inside one cluster in Modified LEACH respectively. So optimum number of cluster head always is vital for network lifetime. From the simulation we can see, in Modified LEACH can improve network performance based of cluster head.

\subsubsection{Energy Consumption Simulation}

The comparison of energy consumption with node is shown in Fig. 7, where energy consumption by LEACH protocol is more than Modified LEACH protocol. Therefore the energy figures illustrates that Modified LEACH protocol reduces the energy consumption.

\subsubsection{Data Received by Base Station}

Fig. 8 shows the comparison between the amounts of data received by base station with time. There are effective broadcast by clusterhead, because of the effective data transmission fail means retransmission is needed that will send more data to the base station. Here the simulation has been done upto 50s and clusterhead stop sending data until the transmitted data reached at destination. Our protocol, Modified LEACH protocol under optimum number of clusterhead can improve efficiency of the wireless sensor networks.

\section{SIMULATION RESULT}

LEACH uses broadcasting with multi-hop delivery to send the packets to the whole network which will follow all possible routes to its destination until the massage sent successfully. In Fig 3 and Fig 4 shows the cluster formation phase and cluster head broadcasting massage to the neighbor clusterhead or base station. In Fig 5 and Fig 6 shows visualization of packet broadcast in TinyViz at runtime simulation. The graphical display showed nodes are periodically becomes clusterhead and broadcasting packets. Here in LEACH it is seen packets were being sent to a random to other node within 
range and the purple color lines are not continuous, indicates the number of retransmission increasing where as in Modified LEACH continuous broadcasting takes place till packet reached at destination. Table I shows the comparison of dead nodes for both the routing protocols where it is seen in Modified LEACH the number of dead node is less than $\mathrm{LEACH}$, indicates Modified LEACH will extend lifetime of the network than LEACH.

task void advertise 0

\{

RoutePacket ${ }^{*} \mathrm{pRP}=\left(\right.$ RoutePacket $\left.{ }^{*}\right)$ \&routeMsg.data[0];

uint8 $\mathrm{t}$ length $=$ sizeof(RoutePacket);

// If busy sending don't send again

if (sendRouteBusy $==$ TRUE)

\{

return;

\}

pRP->addr $=$ addr

atomic addr $=$ TOS BCAST ADDR

pRP $>$ nodeID $=$ TOS_LOCAL_ADDRESS;

pRP-> depth $=$ depth;

pRP $>$ round = round

pRP-sisClusterHead = isClusterHead;

// Set fields for forwarding request

if (pRP->addr != TOS_BCAST_ADDR)

dbg(DBG_TEMP, "MHLeachPSM - Requesting node \%i become a cluster head $\backslash n$ ", pRP->add $\mathrm{pRP}>>$ becomeClusterHead = TRUE;

\}

else

pRP-sbecomeClusterHead = FALSE;

if (call SendMsg.send(TOS_BCAST_ADDR, length, \&routeMsg) == SUCCESS)

Fig 1: Requesting neighbor to become a clusterhead in

LEACH

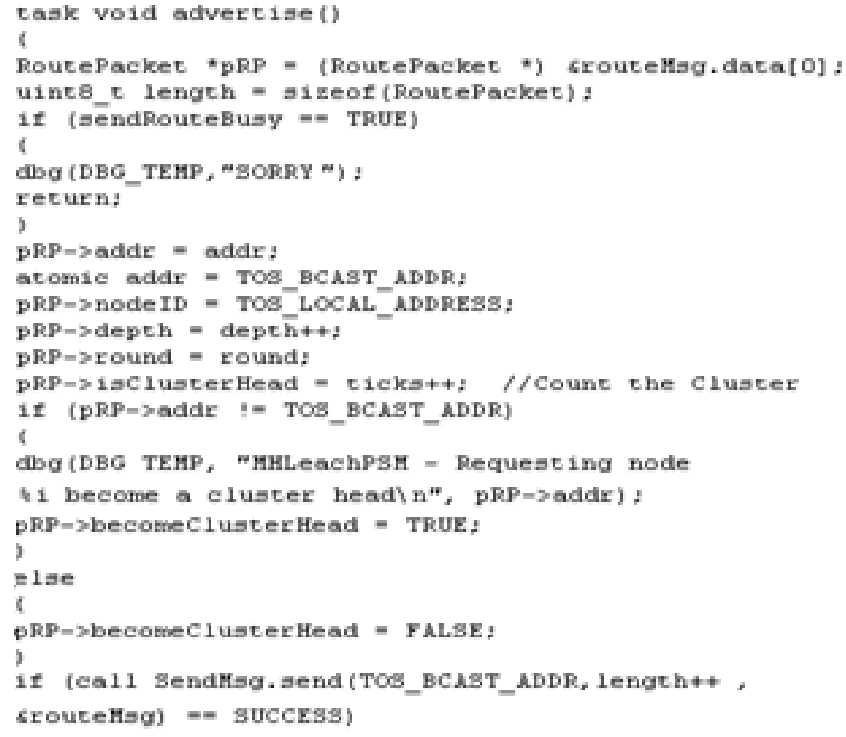

Fig 2: Requesting neighbor to become a clusterhead in Modified LEACH

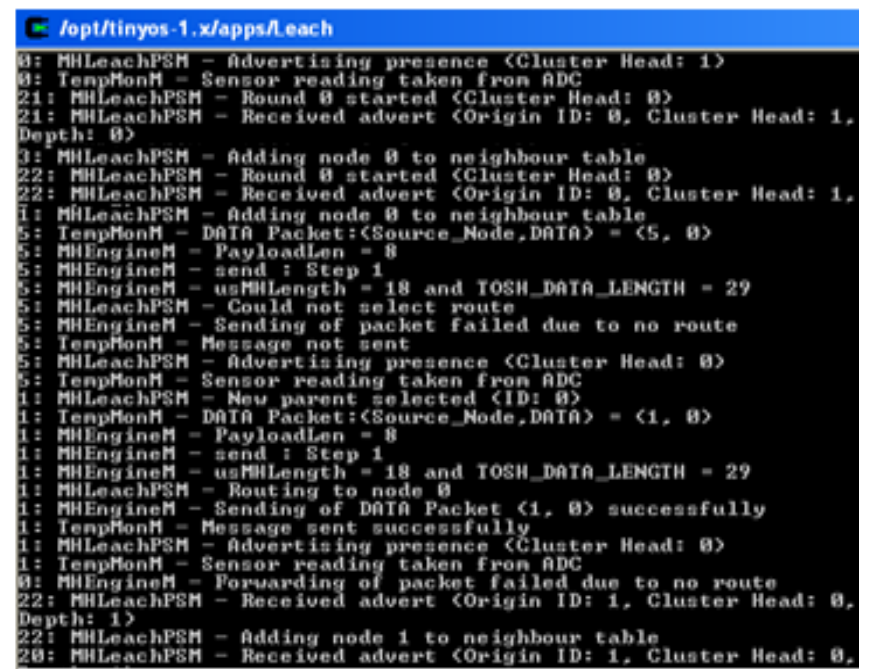

Fig 3: Cluster formation and clusterhead send data in LEACH

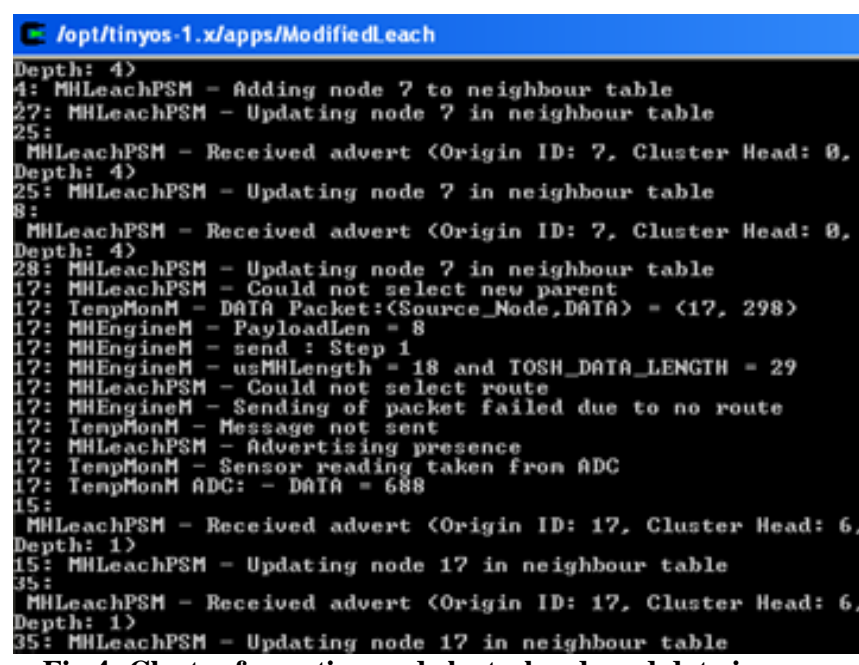

Fig 4: Cluster formation and clusterhead send data in Modified LEACH

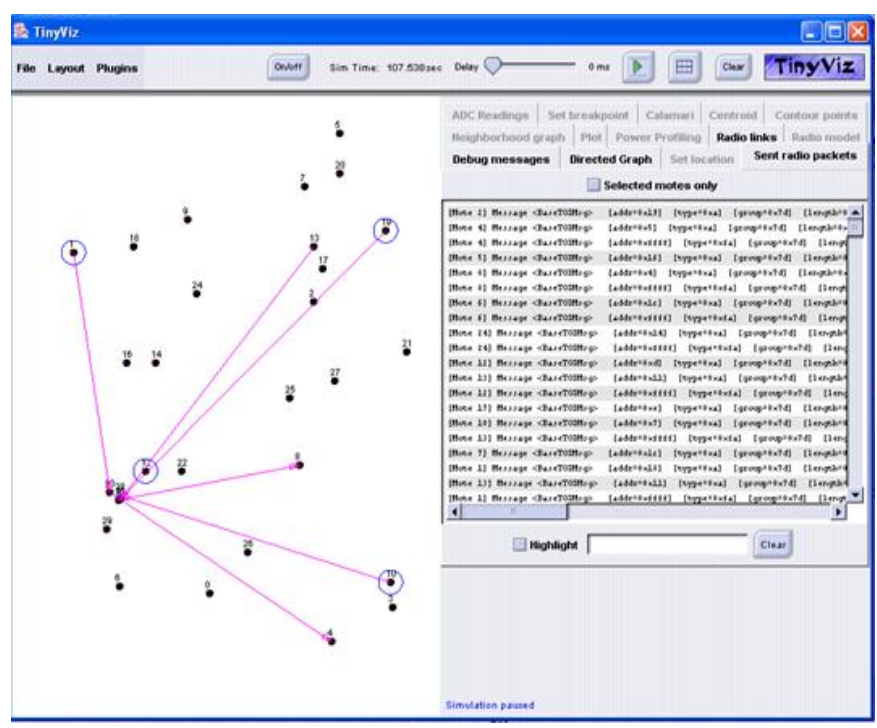

Fig 5: LEACH protocol massage broadcast displayed using Tiny Viz. 


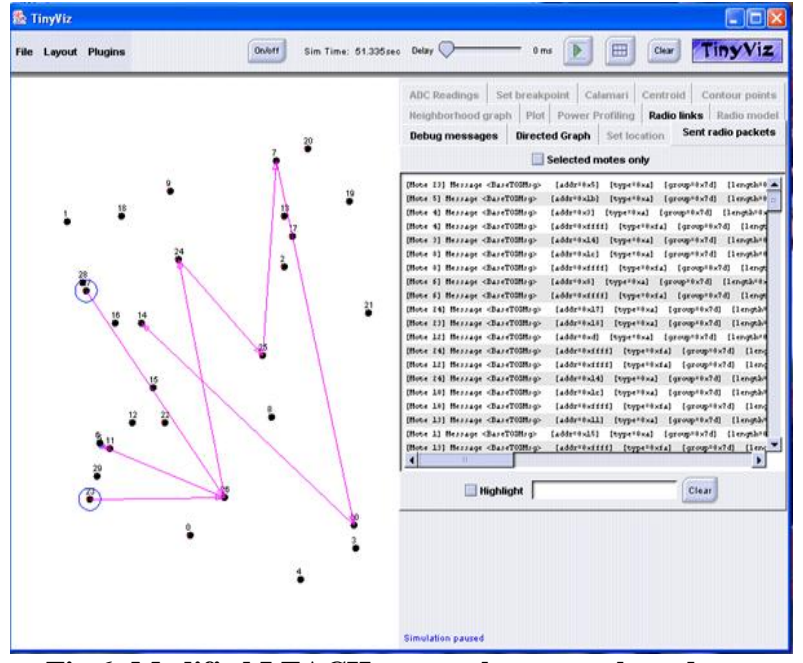

Fig 6: Modified LEACH protocol massage broadcast displayed using TinyViz

\begin{tabular}{|c|c|c|c|}
\hline $\begin{array}{c}\text { No of Sensor } \\
\text { Nodes }\end{array}$ & Time(Sec.) & Dead Node(Leach) & $\begin{array}{c}\text { Dead } \\
\text { Node(Modified } \\
\text { Leach) }\end{array}$ \\
\hline 100 & 300 & 27 & 40 \\
\hline 100 & 330 & 40 & 52 \\
\hline 100 & 350 & 62 & 54 \\
\hline 100 & 380 & 74 & 60 \\
\hline 100 & 400 & 80 & 71 \\
\hline 100 & 430 & 89 & 84 \\
\hline 100 & 450 & 96 & 94 \\
\hline 100 & 500 & 100 & 94 \\
\hline 100 & 550 & 100 & 100 \\
\hline
\end{tabular}

Table I: Simulated result in terms of dead node for both the protocols.

\section{PERFORMANCE ANALYSIS}

Extensive simulation carried out by using TOSSIM for both LEACH and Modified LEACH. In Fig.7 compares energy consumption with increasing on nodes in the network and shows Modified LEACH prolong system lifetime. Fig. 8 explains node broadcast with time is increasing than LEACH protocol. The result in Fig. 9 shows LEACH performs batter upto certain label but at run time simulation it is seen number of dead node in LEACH is more than Modified LEACH after certain rounds, indicates for long run Modified LEACH will be much more applicable than LEACH.

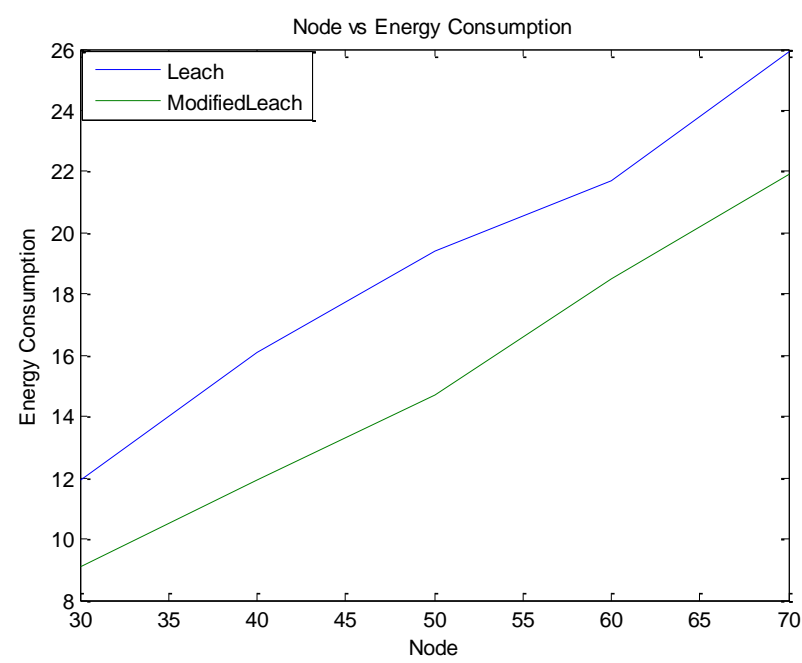

Fig 7: Energy consumption with node for both the protocols

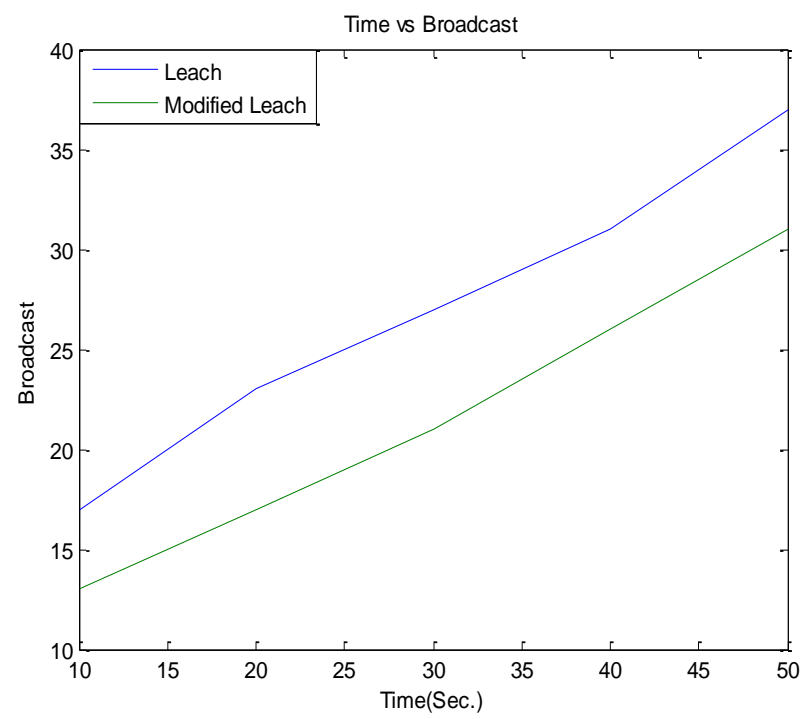

Fig 8: Packet broadcast to the base station by both the protocols

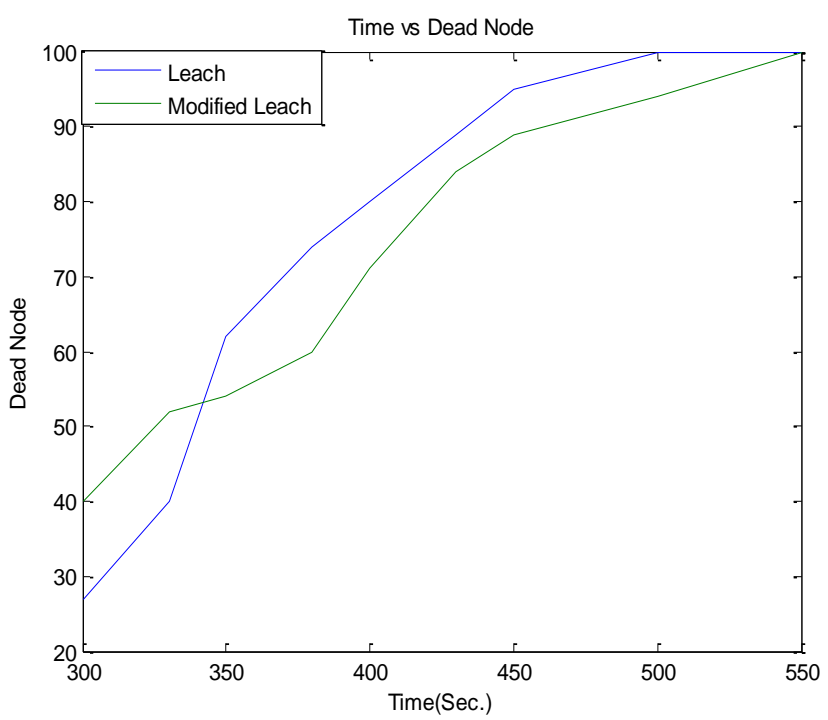

Fig 9: Number of dead node is more in LEACH than Modified LEACH. 


\section{CONCLUSION}

LEACH and Modified LEACH protocols were implemented in TinyOS with some degree of success. The basic evaluation of these protocols was carried out and different phase comparison has been made and showed by different graphs. During the design and implementation of the protocols it was clear that performance gains by Modified LEACH better than $\mathrm{LEACH}$. The implemented protocols might prove to be more successful when used for routing packets in sensor networks.

\section{REFERENCES}

[1] Limin, S., Jianzhong, L. and Yu, C. 2005. Wireless Sensor Networks, Tsinghua publishing company, Beijing.

[2] Akyildiz, I. F. and Y, W. Su. 2002. Sankarasubramaniam, "A Survey on Sensor Networks", IEEE Communications Magazine, 40(8), pp.102-114

[3] Pottie, G. J. and Kaiser, W. J. 2000. "Embedding the Internet: Wireless Intergrated Network Sensors", Communications of the ACM, 43(5), pp.51-58.

[4] Estrin, D. 2002. "Tutorial Wireless Sensor Networks-Part IV: Sensor Network Protocols", MobiCom.

[5] Heinzelman, W., Chandrakasan, A. and Balakrishnan, H. 2000. "Energy-eficient Communication Protocol for
Wireless Sensor Networks", Proceeding of the Hawaii International Conference on System Sciences, Hawaii.

[6] Wei, W. 2006. "Study on Low Energy Grade Routing Protocols of Wireless Sensor Networks", Dissertation, Hang Zhou, Zhe Jiang university.

[7] Xiaoyan, M. 2006. "Study and Design on Cluster Routing Protocols of Wireless Sensor Networks", Dissertation, Hang Zhou, Zhe Jiang university.

[8] Xun-xue, C. 2009. "Wireless Sensor Network Brief Tutorial”, Qing Hua University Press,Beijing.

[9] Mei-hong, H., Cheng-qian, X. and Dong-liang, Z. 2009. "The simulation and analysis of LEACH protocol Based on NS2", Electronic Measurement Technology, vol.1, pp.40-42.

[10] Levis, P. and Lee, N.2003. TOSSIM: A Simulator for TinyOS Networks, Included with the TinyOS 1.1.0 software. 2003.

[11] Gay, D., Levis, P., Behren, R.,Welsh, M., Brewer, E. and Culler, D. 2003. "The nesC Language: A Holistic Approach to Networked Embedded Systems". In Proceedings of Programming Language Design and Implementation (PLDI). 